\title{
Why Cannot BCLC 0- or A-Stage Patients Receive Curative Treatment?
}

\author{
Tse-Ming Kuo $^{a}$ Kai-Ming Chang ${ }^{b}$ Kuo-Jang Kaob \\ aDivision of Gastroenterology and Hepatobiliary Disease, Department of Internal Medicine, \\ Koo Foundation Sun Yat-Sen Cancer Center, Taipei, Taiwan, ROC; b Department of Research, \\ Koo Foundation Sun Yat-Sen Cancer Center, Taipei, Taiwan, ROC
}

\author{
Keywords \\ Hepatocellular carcinoma - Early stages - Barcelona Clinic Liver Cancer staging - Curative \\ treatment $\cdot$ Transcatheter arterial chemoembolization
}

\begin{abstract}
Introduction: Barcelona Clinic Liver Cancer $(B C L C)$ staging has been an important clinical guideline for the management of hepatocellular carcinoma (HCC). BCLC 0 and A stages (BCLC $\mathrm{O} / \mathrm{A}$ ) have been designated as the early-stage HCC, and the curative treatment is recommended as the primary therapeutic modality. However, a recent study indicated that a significant number of BCLC O/A patients were not initially managed with the curative treatment without knowing why. Methods: We, therefore, conducted a study on BCLC 0/A patients who had and had not received initial curative treatment cared at our cancer center from January 2011 to December 2015 and analyzed causes contributing to not having the initial curative treatment. Results: One hundred and sixty-nine BCLC 0/A patients were identified and included in the study. Seventy two patients (43\%) received the initial curative treatment and 97 patients (57\%) did not. After careful review of medical records, all 97 patients without the initial curative treatment had identifiable reasons for not having the initial curative treatment. Two main reasons for not having the initial curative treatment were "probable presence of additional HCC and requiring diagnostic angiography" (28\%) and "difficult or complicating anatomical location of tumors" (17\%). When the relevant clinical parameters were compared between the 2 groups of patients, it was found that patients without the initial curative treatment had more serious clinical conditions and worse overall and recurrence-free survival outcomes compared with those who had the initial curative treatment. Discussion/Conclusion: Our finding indi-
\end{abstract}


cates that a significant fraction of the BCLC 0/A HCC patients is unable to have initial curative treatment as recommended by BCLC guidelines. These early stages of HCC patients represent a distinctive subpopulation and are in need of further investigation to improve their survival outcomes.

(c) 2020 The Author(s)

Published by S. Karger AG, Basel

\section{Introduction}

Hepatocellular carcinoma (HCC) is the fifth most common cancer in men and the ninth most common in women worldwide. Globally, it is the second leading cause of cancer death in men and the sixth leading cause among women $[1,2]$. The majority of HCC patients reside in Eastern Asia. At present, HCC is managed according to Barcelona Clinic Liver Cancer (BCLC) stages using different therapeutic modalities including surgery, local ablation therapy, liver transplantation, transcatheter arterial chemoembolization (TACE), and targeted therapy [3, 4]. Surgery, local ablation therapy with percutaneous ethanol injection (PEI), and radiofrequency ablation (RFA) are regarded as the curative approaches and recommended for BCLC $0 /$ A HCC patients [3]. In practice, many BCLC $0 /$ A patients up to $40 \%$ did not receive the initial curative treatments recommended by the BCLC guidelines [5]. A worse survival outcome was also reported for these patients [5]. Reasons for many of BCLC 0/A patients not undergoing initial curative treatment have not been reported. To investigate the clinical causes of not receiving initial curative treatment for BCLC 0/A HCC, we conducted a study on BCLC 0/A HCC patients. The results are reported herein.

\section{Materials and Methods}

\section{BCLC 0/A HCC Patients}

HCC patients cared at the Koo Foundation Sun Yat-Sen Cancer Center in Taiwan from January 2011 to December 2015 were identified according to ICD-9 and ICD-10 codes. Diagnosis of HCC was based on the American Association for the Study of Liver Disease (AASLD) practice guideline [6], the European Association for the Study of the Liver (EASL) practice guidelines [7], and the consensus-based clinical practice guidelines proposed by the Japan Society of Hepatology (JSH) [8-11]. All patients were staged in accordance with BCLC guidelines. BCLC 0/A patients were identified and included in the study. All medical records up to February 29, 2020, were reviewed.

\section{Initial Curative versus Noncurative Treatment Patients}

Patients who received initial treatment of surgical resection, RFA, or PEI were assigned to the initial curative treatment group. All other patients who received TACE as the initial treatment were assigned to the TACE group. The clinical characteristics of patients of the 2 different groups were listed in Table 1. One patient who had surgical mortality was excluded.

\section{Reasons for No Initial Curative Treatment}

To minimize the bias in identification of reasons for not receiving initial curative treatment, we first empirically established a list of 5 probable reasons. These 5 reasons were (1) presence of unacceptable comorbidity; (2) refusal of curative treatment by patients; (3) anatomical condition of a tumor(s) precluding the curative treatment; (4) eligible for curative treatment but failed to receive the treatment; and (5) other reasons. More detailed specific causes for each reason are provided in Table 2. Some patients had more than one reason recorded, and the overriding reason was used as the primary one for the study.

Clinical Parameters for the Study

The clinical parameters extracted from medical records of patients included size and number of HCC, ECOG performance status, Child-Pugh score, and fibrosis-4 index $[12,13]$. The initial treatment date, the 
Gastrointestinal

Tumors

Table 1. Clinical characteristics of 2 different treatment groups

\begin{tabular}{l|l}
\hline Gastrointest Tumors 2020;7:125-133 \\
\hline DOI: 10.1159/000509824 & $\begin{array}{l}\text { @ 2020 The Author(s). Published by S. Karger AG, Basel } \\
\text { www.karger.com/gat }\end{array}$ \\
\hline
\end{tabular}

Kuo et al.: Curative Treatment in BCLC 0/A

\begin{tabular}{|c|c|c|c|}
\hline & \multicolumn{2}{|l|}{ Treatment groups } & \multirow[t]{2}{*}{$p$ value } \\
\hline & curative treatment & TACE & \\
\hline Patients, $N$ & 71 & 97 & \\
\hline \multicolumn{4}{|l|}{ Age } \\
\hline Mean \pm SD & $55 \pm 12$ & $64 \pm 12$ & $<0.0001^{\mathrm{a}}$ \\
\hline \multicolumn{4}{|l|}{ Sex } \\
\hline Male & 60 & 65 & $0.01^{b}$ \\
\hline Female & 11 & 32 & \\
\hline \multicolumn{4}{|l|}{ BCLC stage } \\
\hline 0 & 22 & 7 & $0.0001^{\mathrm{b}}$ \\
\hline $\mathrm{A}$ & 49 & 90 & \\
\hline \multicolumn{4}{|c|}{ Initial curative treatment } \\
\hline Surgery & 64 & 0 & \\
\hline PEI & 2 & 0 & \\
\hline RFA & 6 & 0 & \\
\hline Tumors, $n$ & & & $0.03^{c}$ \\
\hline 1 & 67 & 80 & 1 vs. $>1$ \\
\hline 2 & 3 & 12 & \\
\hline 3 & 1 & 5 & \\
\hline
\end{tabular}

BCLC, Barcelona Clinic Liver Cancer; TACE, transcatheter arterial chemoembolization; PEI, percutaneous ethanol injection; RFA, radiofrequency ablation. ${ }^{\text {a }}$ The Wilcoxon rank-sum test was used. ${ }^{\text {b }}$ Fisher's exact test was used. ${ }^{c}$ The number of tumors was grouped as 1 and $>1$, and Fisher's exact test was used for statistical comparison.

Table 2. Reasons and corresponding codes for not receiving the initial curative treatment

Reason for not receiving initial curative treatment $\quad$ Code

1. Presence of unacceptable comorbidity

a. Patients of poor cardiovascular performance status $1 \mathrm{a}$

b. High surgical risk according to surgeons and not specified $1 \mathrm{~b}$

c. Presence of other major medical conditions

2. Refusal by the patient $\quad 2$

3. Anatomical and liver condition(s) precluding the initial curative treatment

a. Difficult or complicating anatomical location for the initial curative treatment $3 a$

b. Bilobar distribution of tumors $3 \mathrm{~b}$

c. Probable presence of additional tumor by image study and requiring diagnostic angiography $3 \mathrm{c}$

d. Patients at risk of posttreatment liver failure

4. Eligible for curative treatment but failed to receive the treatment 4

\begin{tabular}{ll}
\hline 5. Other reasons & 5
\end{tabular}

initial treatment modality, the tumor recurrence date, and the last follow-up date or date of death were collected for overall survival and recurrence-free survival. Subsequent treatment modality after the initial treatment was identified. Other clinical variables were collected and are listed in Table 3. One patient who had surgical mortality was not included.

\section{Statistical Analyses}

The aforementioned clinical parameters were analyzed for their association with and without initial curative treatment using either Fisher's exact test (for categorical parameters) or the Wilcoxon rank-sum 
Table 3. Comparison of clinically relevant parameters between the initial curative treatment and the initial TACE groups

\begin{tabular}{|c|c|c|c|}
\hline & $\begin{array}{l}\text { Curative treatment } \\
(N=71)\end{array}$ & $\begin{array}{l}\text { TACE } \\
(N=97)\end{array}$ & $p$ value \\
\hline \multicolumn{4}{|l|}{ Child-Pugh score $^{\mathrm{a}}$} \\
\hline A5 & 66 & 85 & $0.31^{\mathrm{b}}$ \\
\hline A6 & 4 & 9 & \\
\hline B7 & 1 & 3 & \\
\hline \multicolumn{4}{|l|}{ HBs Ag $^{\mathrm{a}}$} \\
\hline Negative & $16(23 \%)$ & $44(45 \%)$ & $0.003^{\mathrm{b}}$ \\
\hline Positive & $54(77 \%)$ & $53(55 \%)$ & \\
\hline Unknown & 1 & & \\
\hline \multicolumn{4}{|l|}{ Anti-HCV ${ }^{\mathrm{a}}$} \\
\hline Negative & $56(84 \%)$ & 67 (69\%) & $0.044^{\mathrm{b}}$ \\
\hline Positive & $11(16 \%)$ & $30(31 \%)$ & \\
\hline Unknown & 4 & & \\
\hline Tumor size, $\mathrm{cm}$ & $2.5 \pm 0.9$ & $2.8 \pm 0.9$ & $0.028^{\mathrm{c}}$ \\
\hline Total bilirubin & $0.69 \pm 0.28$ & $0.82 \pm 0.36$ & $0.032^{c}$ \\
\hline Albumin & $4.4 \pm 0.4$ & $4.1 \pm 0.4$ & $<0.0001^{\mathrm{c}}$ \\
\hline Prothrombin time & $10.9 \pm 0.6$ & $11.3 \pm 0.8$ & $0.0001^{\mathrm{c}}$ \\
\hline Platelet $\left(\times 10^{-3}\right)$ & $184 \pm 58$ & $138 \pm 58$ & $<0.0001^{\mathrm{c}}$ \\
\hline FIB-4 & $1.8 \pm 1.0$ & $4.0 \pm 3.0$ & $<0.0001^{\mathrm{c}}$ \\
\hline AFP & $576 \pm 1,378$ & $852 \pm 2,748$ & $0.121^{\mathrm{c}}$ \\
\hline AST & $37 \pm 20$ & $50 \pm 34$ & $0.001^{\mathrm{c}}$ \\
\hline ALT & $48 \pm 28$ & $54 \pm 40$ & $0.710^{\mathrm{c}}$ \\
\hline
\end{tabular}

TACE, transcatheter arterial chemoembolization; AFP, alpha-fetoprotein; FIB-4, Fibrosis-4. ${ }^{\text {a }}$ The numbers for Child-Pugh score, HBs Ag, and Anti-HCV were numbers of patients. ${ }^{b}$ Compared by Fisher's exact test. ${ }^{\mathrm{c}}$ Compared by the Wilcoxon rank-sum test.

test (for continuous parameters due to violation of the normality assumption). The survival comparison was studied by using the Kaplan-Meier analysis and the log-rank test. Significance level for all statistical tests was set at 0.05. R version 3.5 (www.r-project.org) software was used for the study.

\section{Results}

A total of 169 BCLC 0/A patients were identified and included in the study. Seventy-two patients (43\%) received initial curative treatment including surgical resection, RFA, or PEI. The remaining 97 patients $(57 \%)$ received TACE as the initial treatment. No patient was treated with liver transplantation. A total of 127 patients (75\%) had HCC diagnosis and confirmation by histopathology of biopsy or resected tumor specimens. The remaining 42 patients (25\%) were diagnosed using the noninvasive imaging criteria [6-11]. The clinical characteristics of these 2 different treatment groups of patients are summarized in Table 1.

As shown in Table 1, patients who did not receive the initial curative treatment were older and majority of them were females. In addition, there were relatively more BCLC 0 stage and less BCLC A stage patients in the initial curative treatment group ( $p=0.0001)$. This finding indicated that the initial TACE noncurative treatment group patients had higher BCLC stages than the initial curative treatment group. There was no significant difference in the number of tumors present at the time of initial treatment between the 2 groups of patients.

When we compared the overall survival between these 2 groups of patients, the patients with the initial curative treatment had significantly better recurrence-free and overall survival 
Gastrointestinal

Tumors

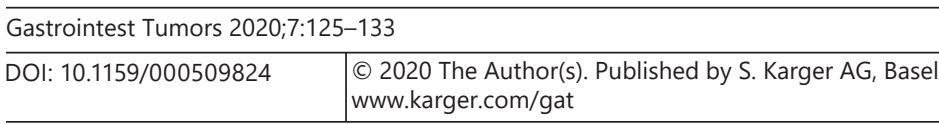

Kuo et al.: Curative Treatment in BCLC 0/A
Fig. 1. Comparison of recurrencefree and overall survival between BCLC 0/A patients who had and had not received the initial curative treatment. There are 71 patients in the initial curative treatment group and 97 patients in the noncurative treatment TACE group. The result showed that patients of the initial curative treatment group had significantly better recurrence-free and overall survival than those of the TACE group $(p<0.0001)$. BCLC, Barcelona Clinic Liver Cancer; TACE, transcatheter arterial chemoembolization.
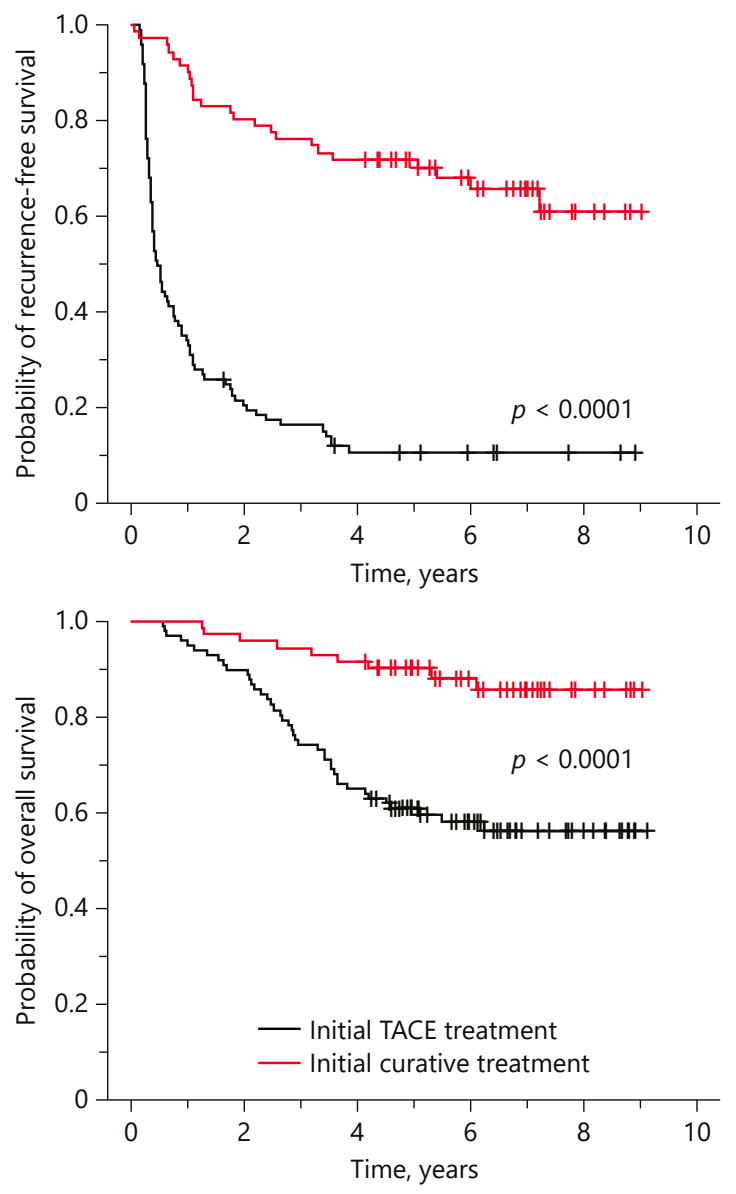

than the patients without the initial curative treatment (Fig. 1). To understand how clinical status differed between these 2 groups of patients, clinical parameters listed in Table 3 were compared. As shown in Table 3, the initial curative treatment group had significantly better values for all the listed clinical parameters except alpha-fetoprotein (AFP) and ALT. Among the parameters compared, serum albumin level, platelet count, and Fibrosis-4 (FIB-4) index showed the most significant difference between these 2 groups. The findings indicated that patients in the initial TACE group had worse clinical conditions associated with their liver disease. When we compared the recurrence-free and the overall survival of these 2 groups of patients, the group of patients with the initial curative treatment had significantly better recurrence-free and overall survival (Fig. 1). It was likely that the worse hepatic function could have contributed in part to the worse survival outcome of the initial TACE group patients.

Through the review of medical records, we identified the major causes leading to not heaving the initial curative treatment in patients of the initial TACE group. The causes were coded according to Table 2. As shown in Figure 2, the most common cause was "3c. Probable presence of additional tumor by imaging study and requiring a diagnostic angiography" (28\%). The second common cause was " 3 a. Difficult or complicating anatomical location for the initial curative treatment" (17\%). The third common cause was "1b. High surgical risk 
Gastrointestinal

Tumors
Fig. 2. Relative frequencies of different primary reasons for not receiving the initial curative treatment in BCLC $0 / \mathrm{A}$ patients $(n=$ 97). Eleven out of 97 patients have additional secondary reasons. Only the primary reasons were used to calculate the relative frequencies in percentage for having the initial noncurative TACE treatment. The codes for reasons not receiving the initial curative treatment are shown in the outer pie chart, and the relative frequencies in percentages are shown in the inner pie chart. The definitions of codes for reasons not receiving the initial curative treatment are available in Table 2. BCLC, Barcelona Clinic Liver Cancer; TACE, transcatheter arterial chemoembolization.

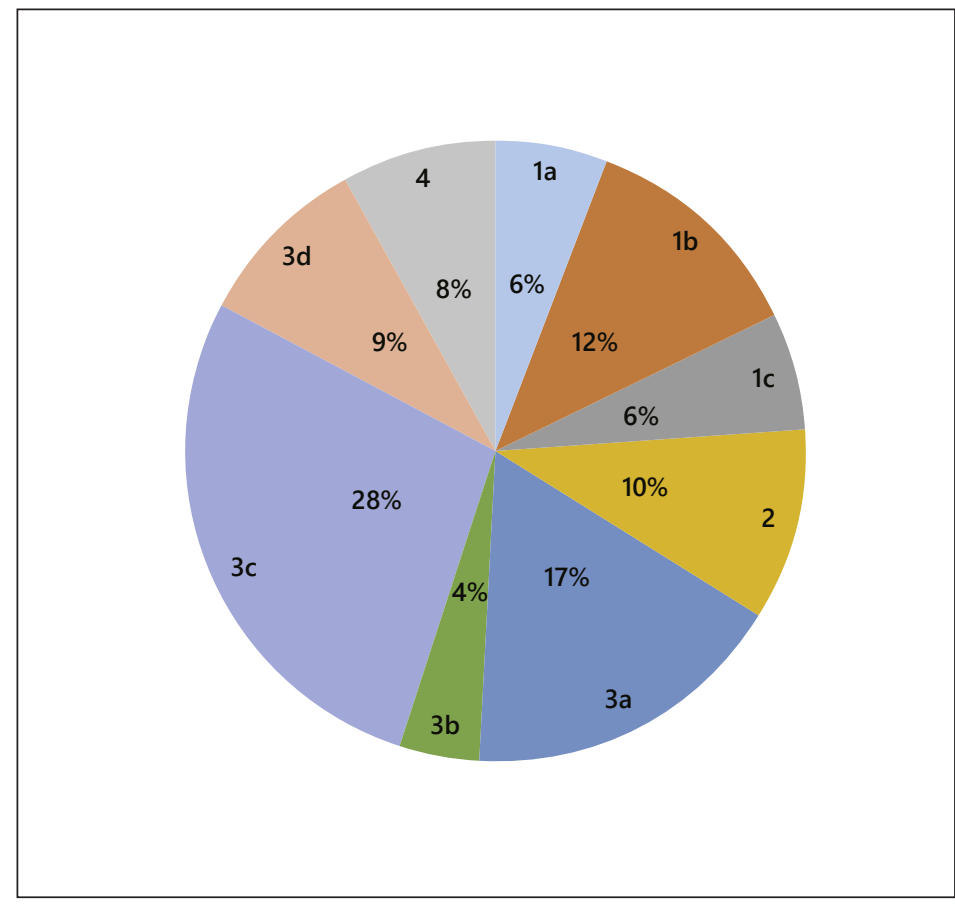

according to surgeons and not specified" (12\%). Other less common ones were "2. Refusal by patients" (10\%), "3d. Patients at risk of posttreatment liver failure" (9\%), "4. Eligible for curative treatment but failed to receive the treatment" (8\%), "1a. Poor cardiovascular performance" (6\%), "1c. Presence of other major medical conditions" (6\%), and "3b. Bilobar distribution of tumors" (4\%).

In the initial TACE group patients, 35 patients had additional nondiagnostic lesions by MRI or CT and underwent interventional angiography for diagnosis. These patients were given TACE treatment during the angiographical session because the situation enabled such a concurrent treatment. All 35 patients included in our study did not have $>3$ HCC tumors after angiography. Among them, 8 patients subsequently received the curative treatment of RFA $(n=6)$ or surgical resection $(n=2)$ within an average of $166 \pm 66$ days (mean \pm SD) after the initial TACE therapy. These 8 patients were regarded as eligible for the initial curative treatment and classified as "4. Eligible for curative treatment but failed to receive the treatment." After exclusion of these 8 patients, the remaining 27 patients did not have any subsequent curative treatment and were classified as no initial curative treatment for the reason of category $3 \mathrm{c}$ of "probable presence of additional tumor by image study and requiring diagnostic angiography." If the 8 patients in category 4 had been excluded from the initial TACE group, the remaining no initial curative treatment patients $(n=89)$ still accounted for $53 \%$ of all BCLC $0 /$ A patients.

\section{Discussion}

In our study of 169 BCLC 0/A HCC patients, $57 \%$ of them did not receive the initial curative treatment. Our result confirmed an earlier report that a significant fraction of BCLC $0 /$ A patients $(40 \%)$ did not receive the initial curative treatment as recommended in the BCLC guidelines [5]. Our investigation of the reasons contributing to not having the initial 
curative treatment in these patients revealed that the most common reason was "probable presence of additional tumor by imaging study and requiring diagnostic angiography." This reason accounted for $28 \%$ of patients without receiving the initial curative treatment. The large number of patients not having the initial curative treatment due to this reason is not unexpected because many HCC patients often have concomitant presence of dysplastic nodules or small borderline lesions. These lesions were frequently picked up by abdominal sonography. Although dynamic CT or MRI study can be used to differentiate these small lesions as malignant or nonmalignant nodule, the results often remain nondiagnostic after the CT or MRI study. Contrast-enhanced ultrasound by "Sonazoid" could have helped to some extent, but it was not available in Taiwan until 2017. Tissue diagnosis is the most reliable method for a definitive diagnosis, but liver biopsy is technically challenging and not practical for small liver nodules detected in imaging studies.

The second common reason for not having the initial curative treatment was " 3 a. Difficult or complicating anatomical location for the initial curative treatment" which accounted for $17 \%$ of the initial TACE patients (Fig. 2). Earlier, it was reported that local ablation therapy usually is not feasible or contraindicated when HCC appears as inconspicuous tumor nodules, exophytic or superficially located tumors, or close to important organs (e.g., gallbladder, major blood vessels, or bile ducts) [5]. Although laparoscopyassisted radiofrequency ablation may be considered, HCC close to a large vessel or an extrahepatic organ $(\leq 5 \mathrm{~mm})$ is still regarded as high risk for such a radiofrequency ablation [14]. For laparoscopic hepatectomy, most surgeons consider that lesions located in the posterior or superior part of the liver (segments 1, 7, and 8, and superior part of segment 4) are not easily resectable due to a limited visualization of tumor and difficulty of control bleeding [15]. Therefore, $17 \%$ of cases without having the initial curative treatment for the 3 a reason can be expected.

When we compared the recurrence-free and overall survival between the 2 groups of patients, striking differences were noted (Fig. 1). This finding is consistent with the earlier reported study [5]. Further comparison of clinical parameters between the 2 groups of patients showed that the group without having the initial curative treatment has a worse overall liver function status and clinical condition. Their worse liver function could have contributed in part to the exclusion from receiving the initial curative treatment and the worse survival outcome of the initial TACE group.

Nevertheless, our study also raises a question of whether the initial curative treatment approach per se had contributed to the improved survival outcome in the initial curative treatment group. This is a clinically relevant and important question. Although the earlier studies [16-19] suggest that TACE can be noninferior to the curative treatment, the result of our study is unable to support or refute the conclusion of these earlier studies. A definitive answer to this question can be obtained only through the prospective study of randomizing the category $3 \mathrm{c}$ patients (Table 2 ) into either an initial curative treatment arm or an initial TACE arm.

In conclusion, the results of our study show that approximately $50 \%$ of BCLC $0 /$ A HCC patients did not receive the initial curative treatment for various reasons. The patients without having the initial curative treatment had more serious compromise of their liver function and a worse overall survival outcome. The results of our study together with the earlier study of Roberts et al. [5] indicate that there are 2 distinctive groups of BCLC 0/A HCC patients. Close to $50 \%$ of BCLC 0/A HCC patients cannot be managed with the initial curative treatment as recommended by the BCLC guidelines. These early-stage HCC patients who cannot have the initial curative treatment need to be further studied for improvement of their survival. 


\section{Acknowledgement}

The authors wish to thank Ms. An-Chen Feng and Ms. Christina Lin for their help in identification of the eligible patients.

\section{Statement of Ethics}

This study was approved by the Institution Review Board (ID No. 20190819A) of Koo Foundation Sun Yat-Sen Cancer Center. The use of informed consent was exempted because the study was a retrospective review of medical records and the data were deidentified after collection. This study was conducted in accordance with the ethical standards of the 1964 Declaration of Helsinki and its later amendments.

\section{Conflict of Interest Statement}

The author declares no conflicts of interest.

\section{Funding Sources}

There is no specific funding for the study. The study was conducted as employees of the Koo Foundation Sun Yat-Sen Cancer Center.

\section{Author Contributions}

Tse-Ming Kuo, MD, is responsible for the study design, review of medical records, data collection and interpretation, and manuscript writing. Kai-Ming Chang is responsible for the statistical analyses of the study. Kuo-Jang Kao, MD, PhD, is responsible for the study design, preparation of figures, data interpretation, and manuscript writing.

\section{References}

1 Torre LA, Bray F, Siegel RL, Ferlay J, Lortet-Tieulent J, Jemal A. Global cancer statistics, 2012. CA Cancer J Clin. 2015;65(2):87-108.

2 Ferlay J, Soerjomataram I, Dikshit R, Eser S, Mathers, C, M Rebelo, et al. Cancer incidence and mortality worldwide: sources, methods and major patterns in GLOBOCAN 2012. Int J Cancer. 2015;136(5):E359-86.

3 Forner A, Llovet JM, Bruix J. Hepatocellular carcinoma. Lancet. 2012;379(9822):1245-55.

4 Ayuso C, Rimola J, Vilana R, Burrel M, Darnell A, García-Criado Á, et al. Diagnosis and staging of hepatocellular carcinoma (HCC): current guidelines. Eur J Radiol. 2018;101:72-81.

5 Roberts SK, Gazzola A, Lubel J, Gow P, Bell S, Nicoll A, et al. Treatment choice for early-stage hepatocellular carcinoma in real-world practice: impact of treatment stage migration to transarterial chemoembolization and treatment response on survival. Scand J Gastroenterol. 2018;53(10-11):1368-75.

6 Bruix J, Sherman M. Management of hepatocellular carcinoma: an update. Hepatology. 2011;53(3):1020-2.

7 European Association for the Study of the Liver. EASL clinical practice guidelines: management of hepatocellular carcinoma. J Hepatol. 2018;69:182-236.

8 Kudo M, Matsui O, Izumi N, Iijima H, Kadoya M, Imai Y, et al. JSH consensus-based clinical practice guidelines for the management of hepatocellular carcinoma: 2014 update by the Liver Cancer Study Group of Japan. Liver Cancer. 2014;3(3-4):458-68.

9 Kudo M, Izumi N, Kokudo N, Matsui O, Sakamoto M, Nakashima O, et al. Management of hepatocellular carcinoma in Japan: consensus-based clinical practice guidelines proposed by the Japan Society of Hepatology (JSH) 2010 updated version. Dig Dis. 2011;29(3):339-64.

10 Kudo M, Matsui O, Izumi N, Iijima H, Kadoya M, Imai Y, et al. Surveillance and diagnostic algorithm for hepatocellular carcinoma proposed by the liver cancer study group of Japan: 2014 update. Oncology. 2014;87(Suppl 1):7-21.

11 Kudo M. Early hepatocellular carcinoma: definition and diagnosis. Liver Cancer. 2013;2(2):69-72.

12 Sterling RK, Lissen E, Clumeck N. Sola R, Correa MC, Montaner J, et al. Development of a simple noninvasive index to predict significant fibrosis in patients with HIV/HCV coinfection. Hepatology. 2006;43(6):1317-25. 
13 Tapper EB, Lok AS-F. Use of liver imaging and biopsy in clinical practice. N Engl J Med. 2017;377(8):756-68.

14 Teratani T, Yoshida H, Shiina S, Obi S, Sato S, Tateishi R, et al. Radiofrequency ablation for hepatocellular carcinoma in so-called high-risk locations. Hepatology. 2006;43(5):1101-8.

15 Casaccia M, Andorno E, Domenico SD, Nardi I, Bottino G, Gelli M, et al. Laparoscopic liver resection for hepatocellular carcinoma in cirrhotic patients. Feasibility of nonanatomic resection in difficult tumor locations. J Minim Access Surg. 2011;7(4):222-6.

16 Hsu KF, Chu CH, Chan DC, YU JC, Shih ML, Hsieh HF, et al. Superselective transarterial chemoembolization vs hepatic resection for resectable early-stage hepatocellular carcinoma in patients with Child-Pugh class A liver function. Eur J Radiol. 2012;81(3):466-71.

17 Ishikawa K, Chiba T, Y Ooka, Suzuki E, Ogasawara S, Maeda T, et al. Transarterial chemoembolization as a substitute to radiofrequency ablation for treating Barcelona Clinic Liver Cancer Stage 0/A hepatocellular carcinoma. Oncotarget. 2018;9(30):21560-8.

18 Bargellini I, Sacco R, Bozzi E, Bertini M, Ginanni B, Romano A, et al. Transarterial chemoembolization in very early and early-stage hepatocellular carcinoma patients excluded from curative treatment: a prospective cohort study. Eur J Radiol. 2012;81(6):1173-8.

19 Kim AR, Park E, Kwon SY, Park SJ, Kim YJ, Yoo BC, et al. Efficacy and safety of combined radiofrequency ablation with transarterial chemoembolization in patients with Barcelona Clinic Liver Cancer stage A hepatocellular carcinoma ineligible for curative treatment. Korean J Gastroenterol. 2019;73(3):167-76. 